\title{
Development of an Industrial Heat Storage Using High-Temperature PCM-Graphite Composites
}

\author{
Herbert Zondag \\ Department Energy Transition \\ TNO \\ Petten, the Netherlands \\ herbert.zondag@tno.nl \\ Gertjan Herder \\ Department Energy Transition \\ TNO \\ Petten, the Netherlands
}

\author{
Michel van der Pal \\ Department Energy Transition \\ TNO \\ Petten, the Netherlands
}

Simon Smeding

Department Energy Transition TNO

Petten, the Netherlands

\author{
Gerard Elzinga \\ Department Energy Transition \\ TNO \\ Petten, the Netherlands
}

\author{
Robert de Boer \\ Department Energy Transition \\ TNO \\ Petten, the Netherlands
}

\begin{abstract}
A material development study is carried out on high-temperature PCMs with graphite. D-Mannitol and adipic acid are tested as high temperature PCM materials for application in industrial steam systems. The study concludes that adipic acid is easier to use because of better chemical stability and much less supercooling. For conductivity improvement, $20 \%$ wt of graphite was found to be sufficient.
\end{abstract}

Keywords-PCM, industry, D-Mannitol, Adipic acid, graphite

\section{INTRODUCTION}

In the EU industry, over $8000 \mathrm{PJ}$ is used for heating. About a third of the energy is used to run processes with temperatures under $250^{\circ} \mathrm{C}$, often using process steam at $150-250^{\circ} \mathrm{C}$. Reducing the corresponding fossil energy use can be realized with high temperature heat pumps using renewable electricity to produce steam, together with heat storage to buffer fluctuating heat streams, particularly related to batch processes. PCM (Phase Change Material) can store a large amount of heat in a small temperature interval around the melting temperature, making it very interesting for industrial heat storage, particularly in steam systems. However, too low thermal conductivity and long-term material stability of the PCM are critical issues. The present paper reports on the ongoing FlexSteam project, carried out at TNO, aiming to develop cost-effective PCM-heat storage technology as keycomponent for a sustainable industry.

\section{HEAT STORAGE DESIGN}

PCM heat storage design is essentially a compromise between high energy density, high thermal power and low cost. The optimum for the design depends on the individual application. For industrial applications, typically high specific power, high reliability and short payback times are desirable. To ensure reliability and short payback times, it is sensible to base new designs on well-proven technology. In industrial heat technology, shell-and-tube heat exchangers are the default technology for heat exchange, due to simple and robust design. The design principle of such heat exchangers can easily be used for PCM heat storage, by applying the PCM either at the shell-side or at the tube-side of the heat exchanger. In a previous project, the PCM was applied at the shell side, as shown in Fig. 1 and Fig. 2. For the PCM, both RT70 and $\mathrm{MgCl}_{2} \cdot 6 \mathrm{H}_{2} \mathrm{O}$ were tested (Tmelt respectively $70^{\circ} \mathrm{C}$ and $116^{\circ} \mathrm{C}$ ).
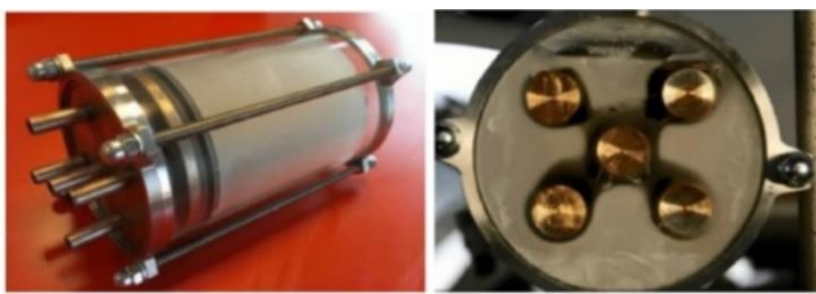

Fig. 1: PCM heat storage lab test setup - PCM applied at shell side.
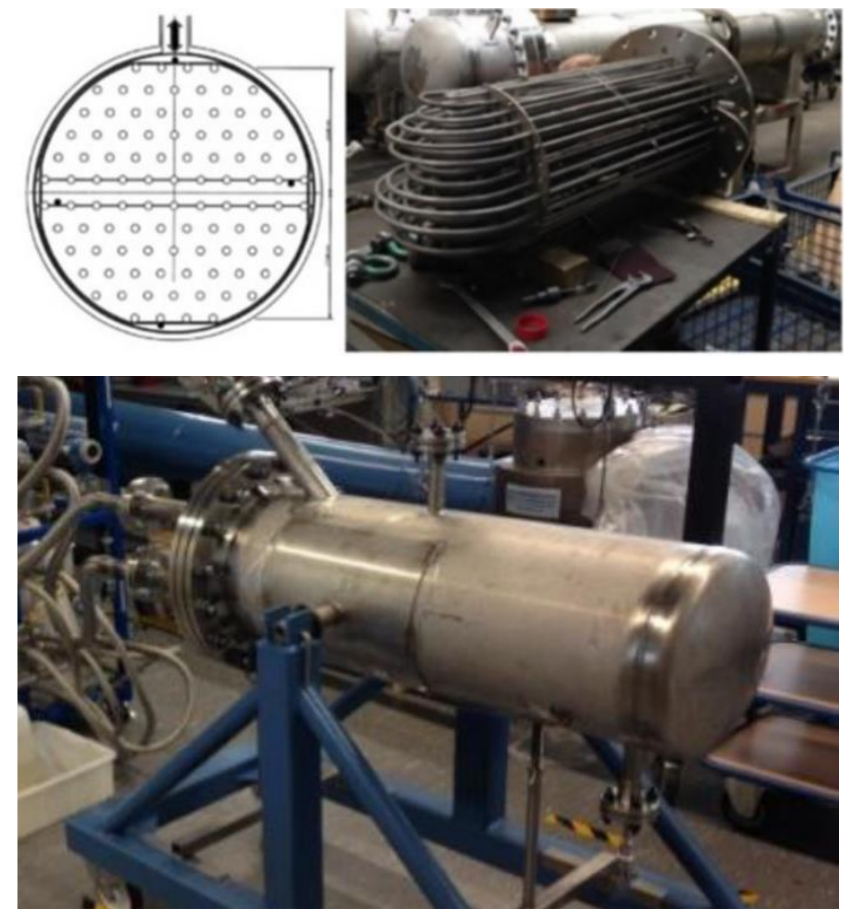

Fig. 2: PCM heat storage prototype - PCM applied at shell side.

1. The low thermal conductivity of the used PCMs was limiting the discharge power of the heat storage. 
2. The large number of heat exchanger tubes and the manufacturing of the hydraulic connections added significantly to the cost of the design.

3. The system was difficult to scale up, and natural convection in the molten PCM caused performance differences between the top side and the bottom side of the PCM storage.

Based on these results, it was concluded that the upscaling potential would be improved by having the PCM inside the tubes; the storage capacity could then be increased relatively easily by adding more tubes, while the hydraulic connections would be avoided, reducing cost. Furthermore, the thermal conductivity of the PCM would have to be increased, allowing for fewer but larger diameter tubes, which would also reduce cost. First tests were carried out on graphite plate impregnated with RT70 and stacked in 1m tubes, that could be cooled or heated via a tube-in-tube construction, with the PCM in the inner tube and the heat transport medium flowing through the spacing between the inner and the outer tube. Although the diameter of the new tube (Fig. 3) was larger than the typical distance between the tubes in the previous configuration (Fig.2), the power was increased from $20 \mathrm{~W} / \mathrm{kgPCM}$ for the old configuration to $130 \mathrm{~W} / \mathrm{kgPCM}$ for the new configuration, which was ascribed to the increase in effective thermal conductivity resulting from the addition of the graphite. Nevertheless, the analysis of the results showed a relatively large internal heat resistance during solidification, lowering the effective interface heat transfer between the PCM and the tube wall from alpha $=175 \mathrm{~W} / \mathrm{m}^{2} \mathrm{~K}$ for the charge phase (liquid in contact with wall) to alpha $=45 \mathrm{~W} / \mathrm{m}^{2} \mathrm{~K}$ for the discharge phase (solid in contact with wall). It was concluded that on solidification, the contact between the PCM-graphite matrix was suboptimal, possibly due to some shrinkage of the PCMgraphite composite, while the graphite matrix would prevent effective adhering of the solidified PCM to the surface (as would have occurred without the graphite).
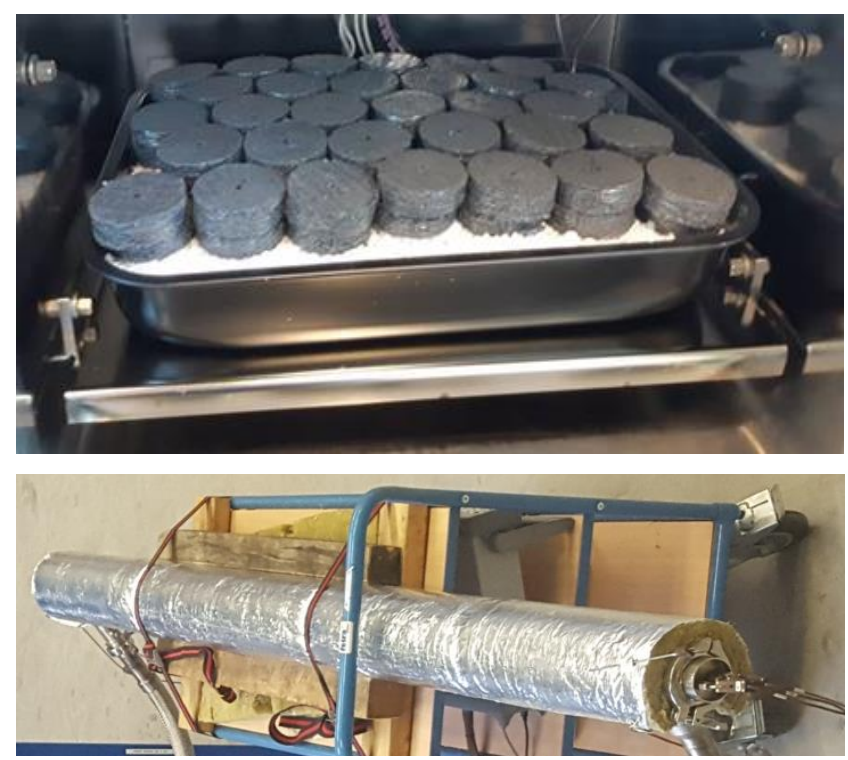

Fig. 3: (a) RT70 impregnated graphite discs, (b) tube-in-tube configuration, with PCM-graphite discs in the inner tube.

\section{REQUIRED PCM CONDUCTIVITY}

For the heat storage to be developed, the aim was set to be able to charge or discharge it completely over a time span of 1 hour, for a temperature difference between heat transfer fluid and $\mathrm{PCM}$ of $10^{\circ} \mathrm{C}$ and a tube diameter of $5 \mathrm{~cm}$. Fig. 4 shows the calculated discharge time for different PCM conductivities, taking into account the thermal resistance between PCM and tube wall as discussed above. Two conclusions can be drawn; (1) the PCM thermal conductivity should be at least $2.5 \mathrm{~W} / \mathrm{mK}$ and preferably $4 \mathrm{~W} / \mathrm{mK}$, beyond that value further improvement in discharge time is limited, (2) the interface resistance is critical and for a value of alpha $=45 \mathrm{~W} / \mathrm{m}^{2} \mathrm{~K}$ it is simply impossible to discharge the tube in 1 hour, whatever the PCM conductivity is. Therefore, it is mandatory to reduce this interface resistance.

The results in Fig. 4 are valid for full discharge; if a lower discharge fraction is acceptable, the discharge time is shortened. Therefore, the fraction of PCM energy content was calculated that would be discharged as a function of discharge time for different values of thermal conductivity and interface heat transfer, as shown in Fig. 5. The figures show that at alpha $=45 \mathrm{~W} / \mathrm{m}^{2} \mathrm{~K}$, in an hour only about $30 \%$ of the energy content is discharged, which is almost independent of the conductivity, since the interface heat transfer is the limiting factor. Only for lower interface resistances, the conductivity has a significant effect on the fraction discharged after 1 hour. Again, this shows that (1) it is crucial to avoid interface resistance (ensure good contact between PCM and wall), preferably keeping the interface heat transfer alpha over $200 \mathrm{~W} / \mathrm{m}^{2} \mathrm{~K}$, (2) the thermal conductivity should be at least $2.5 \mathrm{~W} / \mathrm{mK}$ and preferably $4 \mathrm{~W} / \mathrm{mK}$.

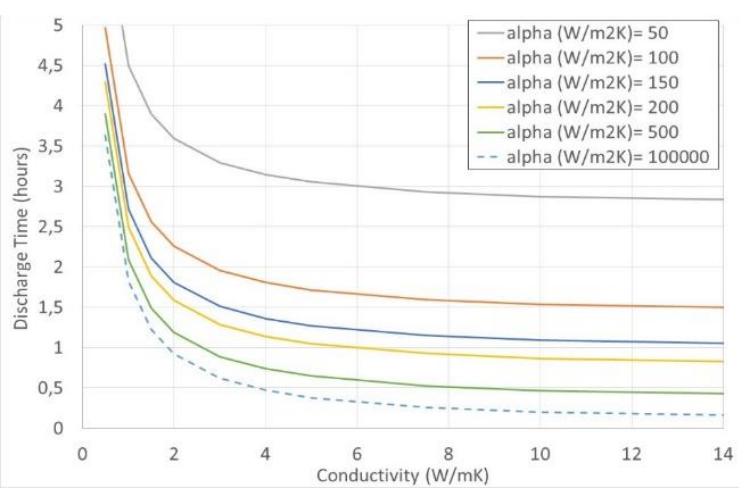

Fig. 4: Discharge time versus effective PCM conductivity for different values of the interface heat transfer coefficient alpha, for PCM in a $5 \mathrm{~cm}$ diameter tube with a temperature difference of $10^{\circ} \mathrm{C}$ between PCM and heat transfer medium. 

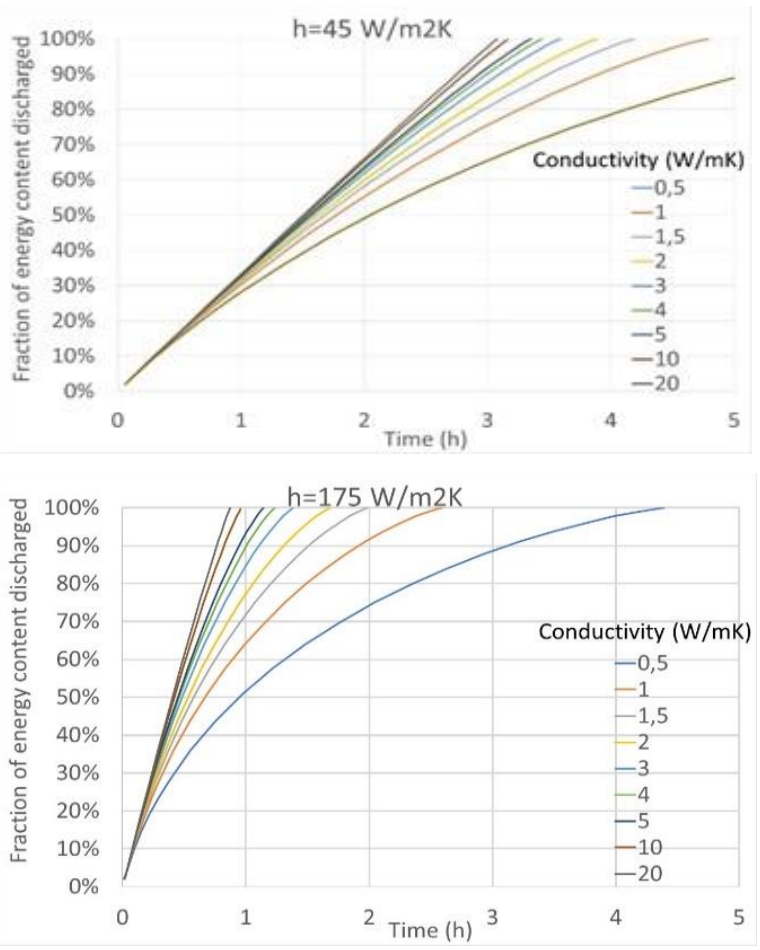

Fig. 5: Fraction of the PCM energy content that can be discharged as a function of time, for different values of thermal conductivity (a) interface heat transfer alpha $=45 \mathrm{~W} / \mathrm{m}^{2} \mathrm{~K},(\mathrm{~b})$ interface heat transfer alpha $=175 \mathrm{~W} / \mathrm{m}^{2} \mathrm{~K}$.

\section{MATERIALS SELECTION}

The aim of producing steam requires PCMs with higher melting temperature than the previously used RT70 and $\mathrm{MgCl}_{2} \cdot 6 \mathrm{H}_{2} \mathrm{O}$. Therefore, new PCM options were investigated. The choice was made to focus on D-Mannitol (a relatively low cost high temperature sugar alcohol, sold under the commercial name Pearlitol 200SD) and on Adipic acid (a low cost dicarboxylic acid, industrially produced at large scale for the manufacturing of Nylon-66). For D-Mannitol and Adipic acid, the thermal conductivity is limited; below $1 \mathrm{~W} / \mathrm{mK}$. Therefore, to reach the required $2.5 \mathrm{~W} / \mathrm{mK}$, conductivity enhancing materials have to be added. Expanded graphite power was chosen, to avoid possible corrosion issues. A check was carried out on the costs of adding graphite, as shown in Table 1. It was concluded that the graphite added significantly to the overall cost, indicating that the fraction of graphite in the composite material should be limited, preferably to $10 \%$ or lower.

\begin{tabular}{|l|c|c|}
\hline & $\begin{array}{c}\text { price } \\
\text { (euro/kg) }\end{array}$ & $\begin{array}{c}\text { energy density } \\
(\mathrm{kJ} / \mathrm{kg})\end{array}$ \\
\hline D-Mannitol & 3,6 & 260 \\
\hline Adipic acid & 0,95 & 250 \\
\hline GFG & 15 & 0 \\
\hline
\end{tabular}

\begin{tabular}{|c|c|c|c|}
\hline & \multicolumn{3}{|c|}{ Cost (euro/MJ) } \\
\hline & 0\%GFG & 10\%GFG & 20\%GFG \\
\hline D-Mannitol & 13,8 & 20,3 & 28,3 \\
\hline Adipic acid & 3,8 & 10,5 & 18,8 \\
\hline
\end{tabular}

Table 1: Cost estimate for PCM with graphite

Graphite can be added to PCM in different ways: (1) expanded graphite plate material may be impregnated with PCM, (2) mixtures of PCM and expanded graphite powder may be pressed into tablets and (3) mixtures of PCM and expanded graphite powder may be molten into a composite material without pressing. The effect of pressing is twofold; a solid graphite structure is realized that remains its integrity even when the PCM melts, resulting in mechanical rigidity, while in addition, the pressing causes orienting of the graphite fibers in such a way that the thermal conductivity of the graphite matrix is enhanced, particularly in the direction perpendicular to the pressing direction. On the other hand, the ease of manufacturing strongly increases from the more complex filling of tubes with impregnating graphite plate to the much easier filling of a tube with a molten mixture of powders. Therefore, it would be convenient if pressing of tablets could be avoided and the tube could be filled directly with the molten mixture. In addition, this may improve contact with the wall during solidification, which is now a limiting factor in the performance.

\section{MATERIALS TESTING}

\section{A. Materials preparation}

Tablets were pressed of D-Mannitol and Adipic acid with $10 \%$ wt of $20 \%$ wt graphite, using three different particle sizes of graphite powder, GFG1200 with particle size distribution $\mathrm{D}_{50}=1200 \mu \mathrm{m}$, GFG600 with particle size distribution $\mathrm{D}_{50}=600$ $\mu \mathrm{m}$ and GFG75 with particle size distribution $\mathrm{D}_{50}=75 \mu \mathrm{m}$. As seen in Fig. 6, the larger particle graphite GFG600 results in a strongly inhomogeneous graphite distribution, while the smaller graphite particles GFG75 give a more homogeneous appearance.

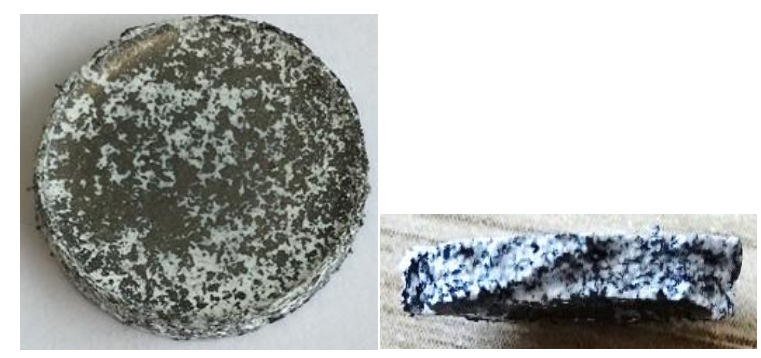

Fig. 6a: D-Mannitol tablet 20\% GFG600 (a) top view, (b) crosssection.

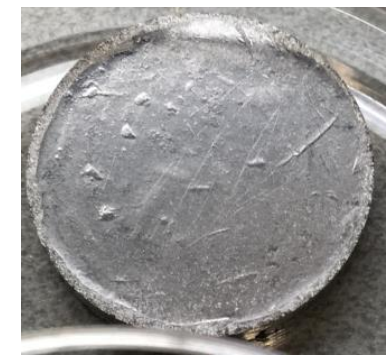

Fig. 6b: D-Mannitol tablet 20\% GFG75

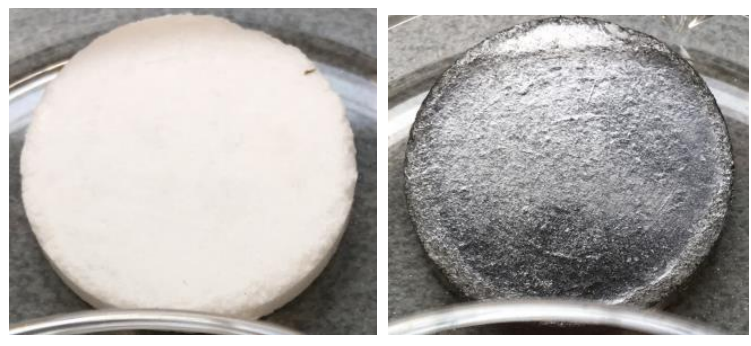

Fig. 7: Adipic acid (a) pure, (b) 20\% GFG75 


\section{B. Thermal conductivity testing}

Next, thermal conductivity tests were carried out by means of laser flash analysis (LFA, Xenon flash) on the compressed PCM-graphite. The results for D-Mannitol for different weight fractions of graphite and different graphite particle sizes are shown in Fig. 8. The conductivity was measured in the direction parallel to the pressing direction, so the direction in which the graphite conductivity is lowest; the numbers presented here should therefore be regarded as minimum values. Fig. 8a clearly shows that with the larger graphite particles GFG600, only limited increase in conductivity was obtained from $0.3 \mathrm{~W} / \mathrm{mK}$ for pure D-Mannitol to $0.6 \mathrm{~W} / \mathrm{mK}$ for D-Mannitol with $20 \%$ GFG600 @ $100^{\circ} \mathrm{C}$. This corresponds to the strongly inhomogeneous distribution of the graphite for this particle size, as seen before in Fig. 6a. The measured thermal conductivity is lower than the required value of 2.5 $\mathrm{W} / \mathrm{mK}$. On the other hand, Fig. 8b shows that with GFG75, a much higher conductivity of $4.5 \mathrm{~W} / \mathrm{mK} @ 100^{\circ} \mathrm{C}$ could be obtained, which is in the required range.

Also, one measurement of the thermal conductivity perpendicular to the pressing direction was carried out (for which the pressed tablet was sliced into parts, that were rotated 90 degrees and put together to make the new "perpendicular" composite sample). As expected from theory and as also clearly seen over here, this leads to a huge increase in the thermal conductivity from $0.6 \mathrm{~W} / \mathrm{mK}$ to $12 \mathrm{~W} / \mathrm{mK}$ for GFG600 (20-fold increase). However, if the pressing of tablets is avoided because of manufacturing feasibility (as argued before), the parallel thermal conductivity is more representative and gives a good estimate for the minimum conductivity that can be expected.

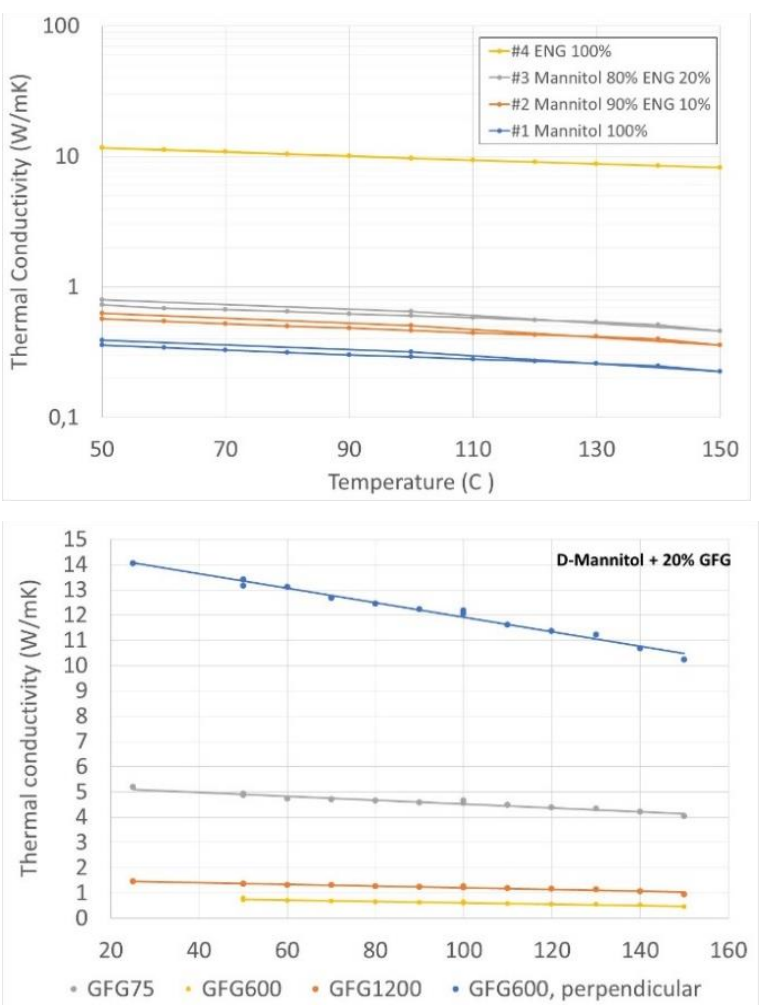

Fig. 8: Thermal conductivity D-Mannitol (a) GFG600, 0-10-20-100\%wt, in pressing direction, (b) GFG 75-600-1200, 20\%wt, in pressing direction \& GFG600 20\%wt, perpendicular to pressing direction.
In Fig. 9, the thermal conductivity for adipic acid is shown with $0 \%, 10 \%$ and $20 \%$ GHG75, increasing the thermal conductivity from $0.9 \mathrm{~W} / \mathrm{mK}$ for pure adipic acid to $5 \mathrm{~W} / \mathrm{mK}$ for $20 \%$ GFG75. In addition, also a test was carried out with a different type of graphite powder GFGHP; adding 20\%wt GFGHP showed an even higher conductivity of $7 \mathrm{~W} / \mathrm{mK}$. It can be concluded that sufficiently high conductivities can be obtained using GFG75 and GFGHP.

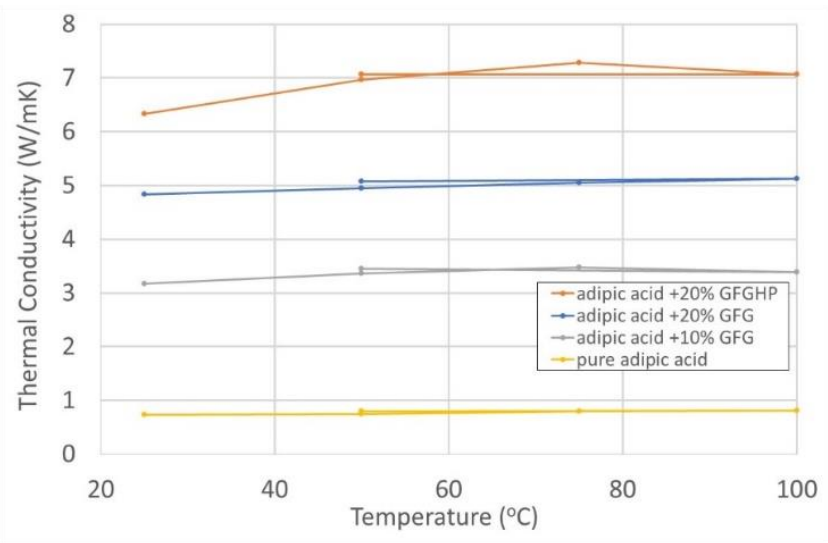

Fig. 9: Thermal conductivity Adipic acid + GFG75 or GFGHP, measured in pressing direction.

\section{DSC testing}

Next, stability of the PCM materials was tested. All tests were carried out in closed cups that had been filled with DMannitol in an oxygen-free $\mathrm{N}_{2}$ atmosphere, since oxygen is known to have a strong degrading effect on D-Mannitol at increased temperatures. Fig. 10 shows the effect of 24 cycles between $100^{\circ} \mathrm{C}$ and $180^{\circ} \mathrm{C}$ on the performance of D-Mannitol (type Pearlitol 200SD). Several observations can be made:

1. The D-Mannitol shows very significant supercooling. This would be strongly disadvantageous for the application to provide industrial steam. However, the use of larger batch sizes is expected to reduce this supercooling. Also, the effect of nucleation agents could be investigated.

2. The D-Mannitol shows polymorphism, as apparent from the double peak appearing in the melting phase. On cooling, the D-Mannitol can crystallize into various lattice structures, that cause different melting points in the subsequent melting phase. The effect of polymorphism is also apparent in the enthalpy plot Fig. 10b, showing incidental dips in the analysis of the melting enthalpy due to badly defined melting peaks. However, the enthalpy in the solidification can be measured more reliably and shows a fairly constant performance.

3. Over cycles, small changes seem to occur in the DSC results; a slight decline in melting temperatures occurs, as well as a more pronounced decline in solidification temperature, as can be observed from the slight difference between the first 5 cycles and the last 5 cycles shown in Fig. 10a. 

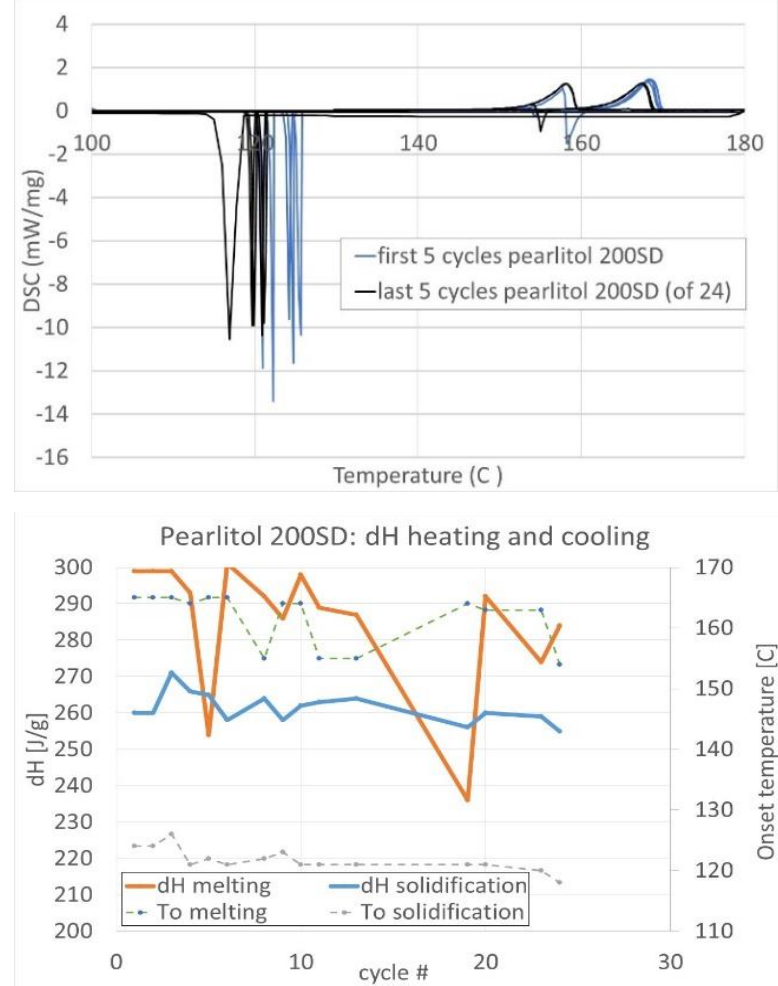

Fig. 10: DSC pure D-Mannitol (pearlitol), 24 cycles (a) DSC power, (b) enthalpy.

In Fig. 11, DSC results (closed cup) are shown for adipic acid, either pure adipic acid or mixed with graphite. Fig. 11a shows that, already in the pure material, supercooling is small, polymorphism does not occur and cyclability is good. The addition of GFG75 does not change the melting temperature, but increases the solidification temperature, which seems to be related to further suppression of the already small supercooling. Finally, Fig. 11c shows the pure adipic acid after 98 cycles; as can be seen no discoloration has occurred, further supporting the conclusion that the sample was chemically stable. This is also seen in Fig. 12, showing a very flat enthalpy curve over the range of cycles.

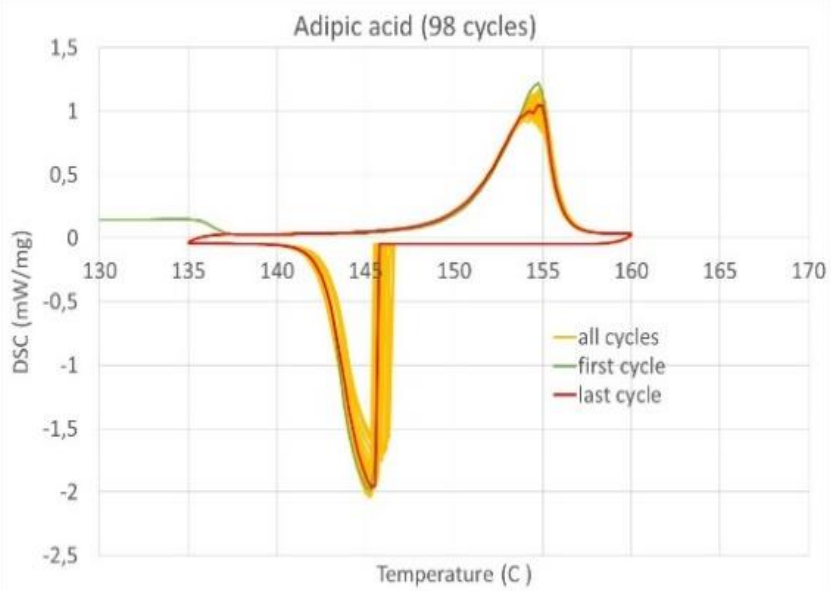

Fig. 11a: DSC results on pure adipic acid, 98 cycles.

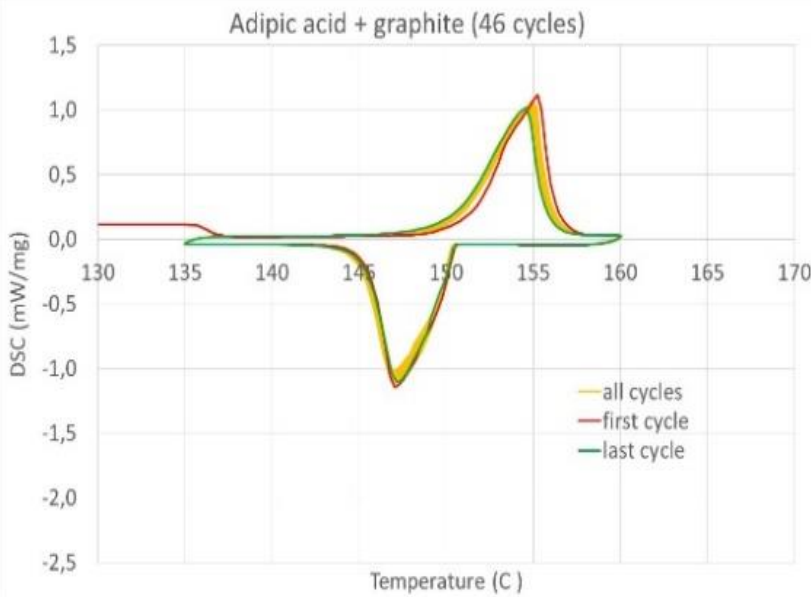

Fig. 11b: DSC results on adipic acid with $20 \%$ GFG75, 46 cycles.

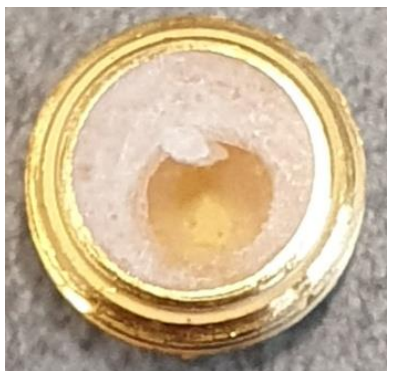

Fig. 11c: DSC cup with pure adipic acid, opened after 98 cycles.
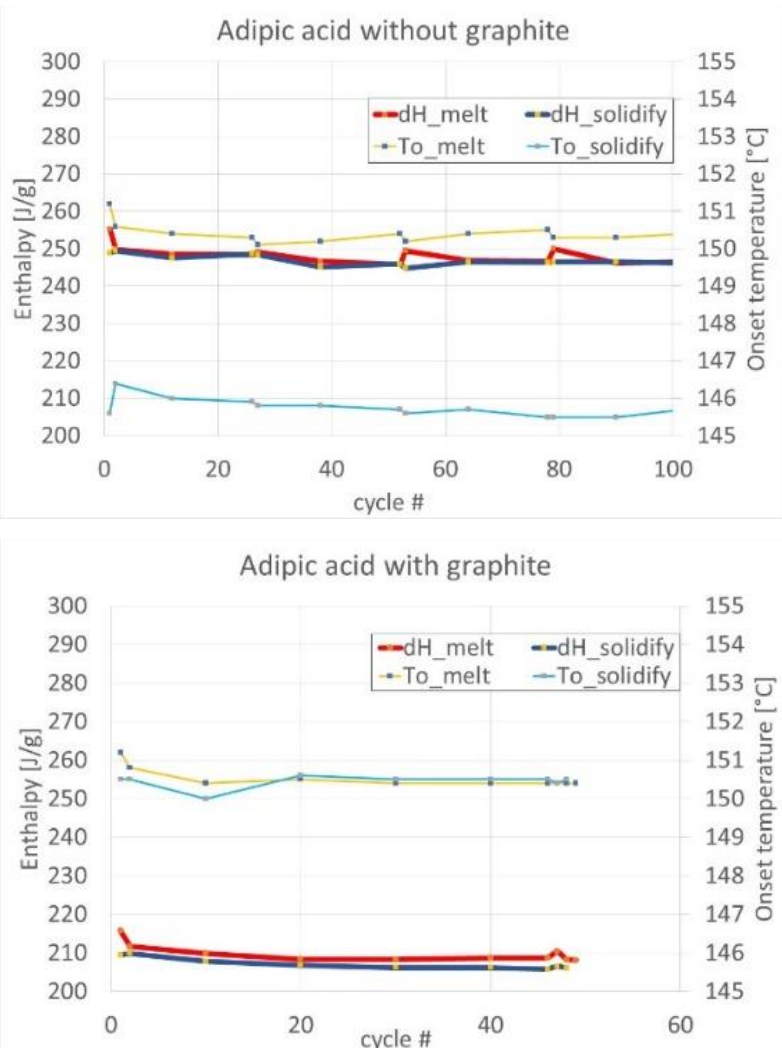

Fig. 12: Melting enthalpy adipic acid (a) pure, 98 cycles, (b) with 20\% GFG75, 46 cycles. 


\section{Thermal cycling setup}

DSC cycling gives information on stability at chemical level, but cannot address issues that occur at larger scale, such as PCM leaking out of matrix material, phase separation or other material redistribution in the sample. Therefore, thermal cycling was carried out on larger scale as well in the thermal cycling lab setup, shown in Fig. 13a. For adipic acid, the thermostatic bath was set to carry out heating/cooling cycles (cycle time about 2.5 hours) between $130^{\circ} \mathrm{C}-170^{\circ} \mathrm{C}$. Four types of samples were tested: (1) tablets with $10 \%$ wt GFG75, (2) tablets with $20 \%$ GFG75, (3) adipic acid powder mixed with $10 \%$ wt GFG75 powder and (4) pure adipic acid as reference. All tubes were covered with aluminum foil to reduce evaporation of the adipic acid, while allowing for some leakage to prevent pressure build-up.

During the testing sequence an error occurred, keeping the thermostatic bath at $170^{\circ} \mathrm{C}$ instead of cycling. This resulted in the samples being at $170^{\circ} \mathrm{C}$ for 4 days, and in addition (after fixing the error) having undergone 28 cycles between $130^{\circ} \mathrm{C}$ and $170^{\circ} \mathrm{C}$. The results are shown in Fig. 13b. The figure shows that a small amount of adipic acid leaked out of the tablet samples and ended up in the empty space at the bottom of the tube below the tablets. The adipic acid itself showed significant yellowing. This may be due to reaction with oxygen leaking in via the aluminum foil, since in the closed cup DSC measurements this effect was not observed (see Fig.11c). Finally, while the tablets showed stable performance, the mixed powder sample showed significant phase separation, with the graphite sinking to the bottom of the sample. This resulted in a stabilized and sharp interface between the GFGdepleted pure adipic acid and the adipic acid that had become concentrated in GFG. This seems to indicate that the graphite could not sink any further, probably due to the volume of the graphite particles. Therefore, using a somewhat higher GFG weight content of e.g. $15 \%$ wt could probably prevent the phase separation.

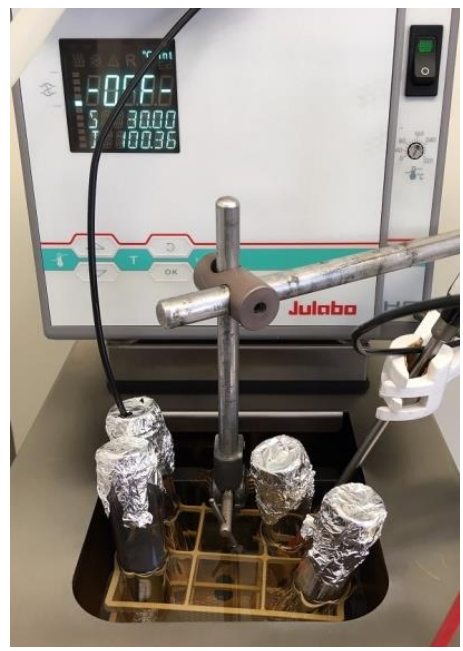

Fig. 13a Thermal cycling setup in thermostatic bath for cycling of adipic acid samples.
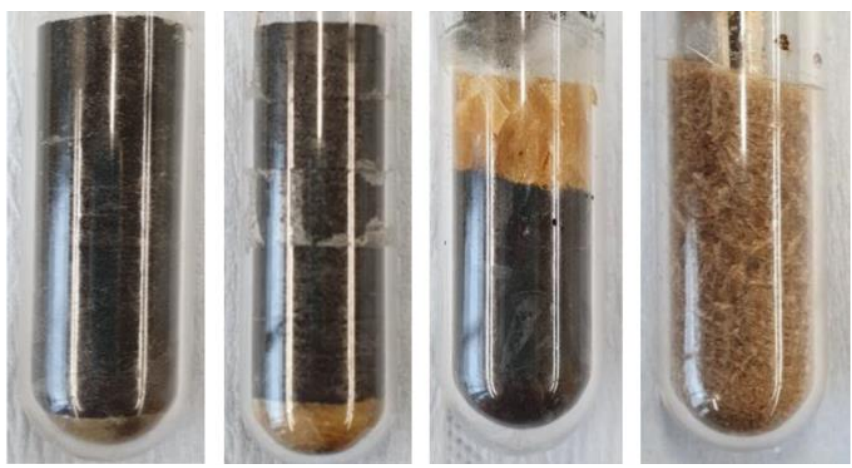

Fig. 13b: Adipic acid samples after cycling: (1) tablets 10\% GFG75, (2) tablets $20 \%$ GFG75, (3) adipic acid \& 10\%wt GFG75, mixed as powder and molten (no tablet pressing), (4) reference (pure adipic acid).

\section{CONCLUSIONS AND FURTHER WORK}

- From the presented results, it is concluded that adipic acid is easier to use than D-Mannitol for industrial steam storage, because of issues in the Mannitol related to supercooling, polymorphism as well as lower chemical stability (as apparent in the cycling tests).

- It was concluded that for the application in tubes, a conductivity of at least $2.5 \mathrm{~W} / \mathrm{mK}$ would be required, as well as good contact with the walls throughout the melting and solidification.

- Finally, it was concluded that addition of $20 \%$ graphite to the PCM could provide sufficiently high thermal conductivity.

For the continuation of the project, this implies that the focus will be on Adipic acid with graphite. Provided that thermal conductivity is high enough, probably a powdery mixture will be applied because of ease of manufacturing.

\section{ACKNOWLEDGMENT}

The authors gratefully acknowledge the contribution of Royal Cosun and SGL, providing the materials used in this project. This project is financially supported by the Topsector Energy subsidy from the Ministry of Economic Affairs and Climate Policy.

\section{REFERENCES}

[1] H.A. Zondag, A.J. Marina, S.F. Smeding, H.S.K. Subramanian, R. de Boer, J. van der Kamp (2019), Experimental results on PCM heat storage with graphite conductivity enhancement, proceedings IRES Conference 2019.

[2] H.A. Zondag, R. de Boer, S.F. Smeding, J. van der Kamp (2018), Performance analysis of industrial PCM heat storage lab prototype, Journal of Energy Storage 18, pp. 402-413. 